\title{
Development, in vitro biocompatibility, and antitumor efficacy of acetic acid-modified Cordyceps sinensis polysaccharide nanoparticle drug delivery system
}

\author{
Jiao Guan ${ }^{1 \#}$, Liqin Han ${ }^{1 \#}$, Nianqiu Shi', Heyun Zhu ${ }^{\circledR 1^{*}}$, Junmin Wang ${ }^{2 *}$ \\ ${ }^{1}$ School of Pharmacy, Jilin Medical University, Jilin City, P. R. China, \\ ${ }^{2}$ Affiliated Hospital, Jinlin Medical University, Jilin City, P. R. China
}

\begin{abstract}
Docetaxel-loaded acetic acid conjugated Cordyceps sinensis polysaccharide (DTX-AA-CSP) nanoparticles were prepared through dialysis and their release rates in vitro, particle sizes, zeta potentials, drug loading capacities, and encapsulation efficiencies were characterized for the synthesis of AA-modified CSPs from traditional Chinese medicine Cordyceps sinensis (Berk.) Sacc. Then, the AA-modified CSPs were characterized by ${ }^{~} \mathrm{H}-\mathrm{NMR}$ and FT-IR. Furthermore, the biocompatibility of the delivery carrier (AA-CSP nanoparticles) was assessed on human umbilical vein endothelial cells. In vitro antitumor activity studies on DTX-AA-CSP nanoparticles were conducted on the human liver (HepG2) and colon cancer cells (SW480). The DTX-AA-CSP nanoparticles were spherical and had an average size of $98.91 \pm 0.29 \mathrm{~nm}$ and zeta potential within the $-19.75 \pm 1.13 \mathrm{mV}$. The encapsulation efficiency and loading capacity were $80.95 \% \pm 0.43 \%$ and $8.09 \% \pm 0.04 \%$, respectively. In vitro, DTX from the DTX-AA-CSP nanoparticles exhibited a sustained release, and the anticancer activities of DTX-AA-CSP nanoparticles against SW480 and HepG2 were significantly higher than those of marketed docetaxel injection (Taxotere ${ }^{\circledR}$ ) in nearly all the tested concentrations. The AA-CSP nanoparticles showed good biocompatibility. This study provided a promising biocompatible delivery system for carrying antitumor drugs for cancer therapy.
\end{abstract}

Keywords: Docetaxel. Cordyceps sinensis polysaccharide. Nanoparticles. Antitumor efficacy. Biocompatibility.

\section{INTRODUCTION}

Anticancer drugs used in tumor treatment are nonselectively target normal and cancer cells and thus often cause toxic side effects. The highly effective delivery of hydrophobic antitumor agents, such as paclitaxel or docetaxel, is often impeded by their poor water solubility, rapid phagocytic and renal clearance, and systemic toxicity. Therefore, studies for reducing the

*Correspondence: H.-Y. Zhu, Department of Pharmaceutical Analysis, School of Pharmacy, Jilin Medical University, 5 Jilin Street, Fengman District, Jilin City, 132013, China. Tel: +86 0432 64560530. Email: zhy19820903@126.com. J.-M.Wang, Department of Internal Neurology, Affiliated Hospital, Jilin Medical University, 81 Huashan Road, Fengman District, Jilin City, 132013, China. Tel: +86 0432 64560535. Email: 1915570346@qq.com

\# These authors contributed equally to this work and are both considered to be the first author toxicities and improving the efficacies of various drug delivery systems, such as self-emulsifying drug delivery systems, liposomes, microspheres, and polymeric micelle nanoparticles, have been conducted (Feeney, 2016; Pakulska, Miersh, Shoichet, 2016; Torchilin, 2014; Pattni, Chupin, Torchilin, 2015).

Recently, micelle nanoparticles that consist of amphiphilic block copolymers and can self-assemble in aqueous media have received great attention as gene and drug nanocarriers because of their special characteristics, such as capability to prolong blood circulation and core-shell structures (Shi, 2010; Shi, 2017; Segal et al., 2017; McHale, 2012). A selfassembled micelle nanoparticle has a unique coreshell backbone composed of a hydrophobic core and hydrophilic shell (Nishikawa, Akiyoshi, Sunamoto, 1996). The monolayer structure can form an outer 
hydrophilic shell and inner hydrophobic core in an aqueous medium through self-assembly, and the special core-shell structure can incorporate and protect drugs in an aqueous medium. The half-time of a self-assembled nanoparticle is prolonged by its special hydrophilic shell during blood circulation; the shell enables the nanoparticle to escape from reticuloendothelial system uptake after intravenous injection (Powers et al., 2017). Amphiphilic block copolymers are usually obtained through the following techniques: chemical crosslinking of monomers, physical self-assembly of macromolecules, and polymerization of monomers in a homogeneous medium (Xu et al., 2015). However, synthetic polymers sometimes exhibit material toxicity, high immunogenicity, and nondegradability, which restrict their application in drug delivery systems. These disadvantages can be offset by using natural polymers (e.g., polysaccharides), which have low immunogenicity, good biocompatibility, and biodegradability and can be easily modified and used to improve the capacities of drug delivery systems (Liao et al., 2005). Pullulan, modified with cholesterol and amino groups as a drug delivery vehicle of anticancer drug (docetaxel), has high antitumor efficacy and strong lung cancer cell inhibition in vitro (Singh, Kaur, Kennedy, 2015). Meanwhile, Kamel et al. (2008) used propylene oxide to modify a cellulose hydroxyl group and prepared hydroxypropyl cellulose to enhance cellulose solubility and control drug release. Chitosan-based, drug-loaded micelles exhibit a remarkably higher inhibition for cancer cells in vitro than reference drugs, which are related to enhance drug internalization (Agnihotri, Mallikarjuna, Aminabhavi, 2004). Cordyceps sinensis (Berk.) Sacc is widely used in China as traditional Chinese medicine (TCM) to improve the kidney, liver, lung, and immune functions. Cordyceps sinensis polysaccharides (CSP) have been widely used in biological areas, such as antioxidation, immunomodulation, and hypoglycemics (Li et al., 2006; Li et al., 2003; Wu et al., 2005; Cheung et al., 2009). CSPs have been considered for various pharmaceutical applications, such as drug delivery systems. Polymeric nanoparticles synthesized by using deoxycholic acid, alkyl, and aralkyl have been used to improve the hydrophobic property of water-soluble copolymers (Lee, 1998). In the present study, amphiphilic polymers with possible antitumor application in pharmaceutical field were obtained by synthesizing acetic acid modified Cordyceps sinensis polysaccharide (AA-
CSP) nanoparticles through a process involving the covalent attachment of AA to polysaccharides through ester links. AA-CSP was characterized with FT-IR spectroscopy and 1H-NMR. Docetaxel (DTX) was selected as a model antitumor drug to prepare DTX-AACSP nanoparticles. The detailed molecular structure of DTX can be found in Figure 1, and the chemical structure and synthetic route of the AA-CSP copolymer were depicted in Figure 2. Polydispersity index, particle sizes, zeta potentials, drug loading capacity (LC), encapsulation efficiency (EE) and in vitro drug release were measured. Biocompatibility assay was evaluated in human umbilical vein endothelial cells (HUVEC) in vitro. Furthermore, in vitro antitumor activity studies of DTX-loaded AA-CSP nanoparticles were performed in the human liver (HepG2) and colon cancer cells (SW480). This study is beneficial to the development of polymer micelle nanoparticles based on TCM and provides a promising delivery system (AACSP nanoparticles) for antitumor drug delivery.

\section{MATERIAL AND METHODS}

\section{Material}

Docetaxel injection (Taxotere $\left.{ }^{\circledR}\right)$ was provided by Sanofi Aventis Pharmaceutical Co., Ltd. (Beijing, China). Methanol and acetonitrile were purchased from Fisher Scientific (Fair Lawn, NJ, USA, chromatographic grade). Docetaxel was provided by Meilun Biological Technology Co., Ltd (Dalian, China, Purity: 99.89\%). Cordyceps sinensis polysaccharides were supplied by Jilin Medical University (Jilin, China, Purity: 90.89\%). Dimethyl sulfoxide (DMSO), pyridine, and acetic anhydride (AA) were supplied by Energy Chemical Co., Ltd. (Shanghai, China). AA was purchased from Sinopharm Chemical Reagent Co., Ltd (Beijing, China). Dulbecco's modified eagle medium (DMEM), fetal bovine serum (FBS), trypsin, and phosphate buffer saline (PBS) were all purchased from Thermo Fisher Scientific (Fair Lawn, NJ, USA). 2K was provided by Wisent Co., Ltd. (Montreal, QC, Canada). Tween 80 , ethanol, diethyl ether and pyridine were supplied by Aladdin Reagent Co., Ltd. (Shanghai, China). Membrane filters were purchased from Tianjin Jinteng Experiment Equipment Co., Ltd (Tianjin, China), and distilled water was supplied by Milli-Q (Germany). All the other reagents were of analytical grade, obtained commercially. 


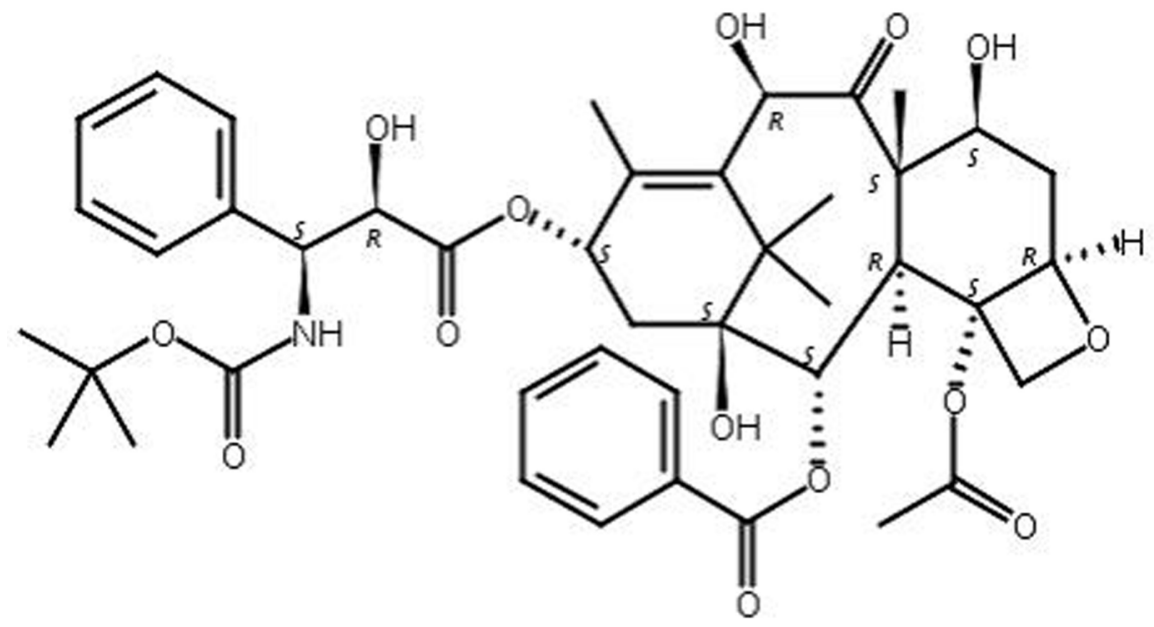

FIGURE 1 - Chemical structure of doxetaxel.

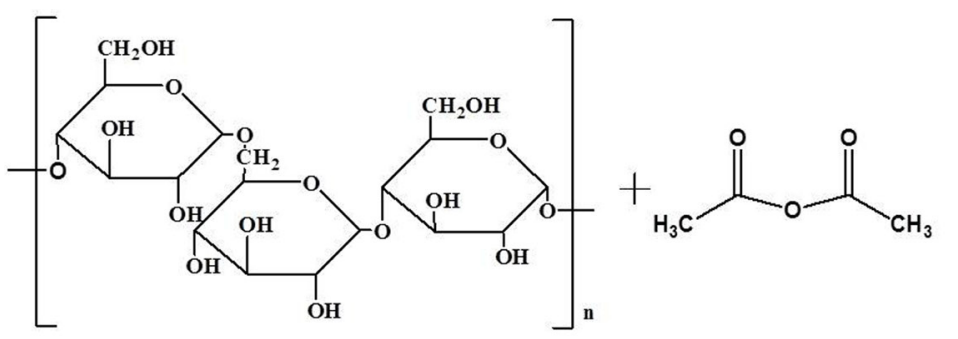

Cordyceps sinensis polysaccharides (CSP)

Acetic anhydride

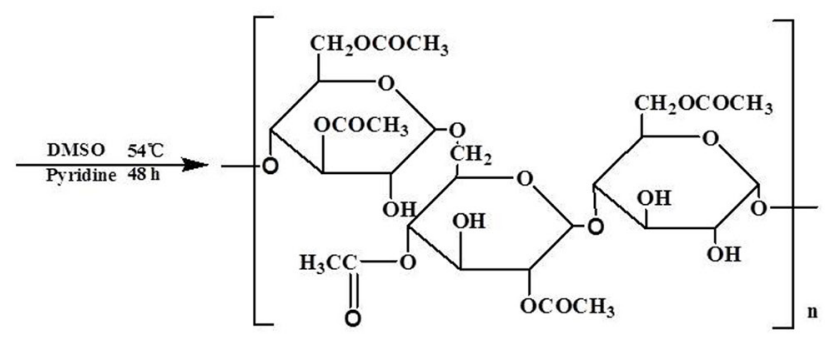

AA conjugated CSP (AA-CSP)

FIGURE 2 - Synthetic route of acetic acid (AA) conjugated Cordyceps. sinensis polysaccharides (AA-CSP).

\section{Synthesis of the AA-CSP Copolymer}

The AA-CSP graft copolymers were synthesized using pyridine, AA, and CSP, as shown in Figure 2. CSP $(2.0 \mathrm{~g})$ was added in $20 \mathrm{~mL}$ of DMSO solution, and then the mixed solution was stirred for $2 \mathrm{~h}$ at $54{ }^{\circ} \mathrm{C}$. Pyridine $(6 \mathrm{~mL})$ and AA $(7.5 \mathrm{~mL})$ were added to the mixed solution $(20 \mathrm{~mL})$ and reacted for $48 \mathrm{~h}$ at $54{ }^{\circ} \mathrm{C}$. The reaction solution was transferred to an excess amount of cold ethanol (10 folds by volume) to obtain precipitates, which were recovered by filtration, washed with ethanol $(100 \mathrm{~mL})$ and diethyl ether $(100 \mathrm{~mL})$ three times, and then dried in vacuum at $54{ }^{\circ} \mathrm{C}$ (Motozato et al., 1986). The AA-CSP copolymer yield was $1.02 \mathrm{~g}$.

\section{FT-IR Spectroscopy}

The changes of the chemical composition in the surfaces of CSP after conjugation with AA were performed on a Thermo IS10 FTIR spectrometer with a tablet of powdered $\mathrm{KBr}$ and adsorbent in the range of 400-4000 $\mathrm{cm}^{-1}$ (Thermo, SA) (Xie et al., 2015).

\section{${ }^{1} \mathrm{H}-\mathrm{NMR}$ Spectroscopy}

The ${ }^{1} \mathrm{H}$ NMR spectra of the samples ( $3 \mathrm{mg}$ ) were determined in $\mathrm{DMSO}_{\mathrm{d} 6}(500 \mu \mathrm{L})$ using a $400 \mathrm{MHz}$ NMR spectrometer (AVIII, Bruker, $400 \mathrm{MHz}$ ) at $25^{\circ} \mathrm{C}(\mathrm{Wu}$, 2007). All the spectra were processed with Bruker Topspin Version 3.0 software. The degree of substitution (DS) of AA-CSP was calculated via ${ }^{1} \mathrm{H}$ NMR.

DS was calculated according to the following equation:

DS $(\%)=\left(\mathrm{A}_{\delta 0.85} / 3\right) /\left(\mathrm{A}_{\delta 5.15}+\mathrm{A}_{\delta 4.91}\right) * 100 \%$,

where A $\delta 0.85$ was the peak area of methyl protons; A $\delta 5.15$ was the peak area of $(1 \rightarrow 6)$-linked hydrogen 
protons; and A $\delta 4.91$ was the peak area of $(1 \rightarrow 4)$-linked hydrogen protons.

\section{Development of DTX-Loaded AA-CSP Nanoparticles}

AA-CSP $(25 \mathrm{mg}$ ) was dissolved in $5 \mathrm{~mL}$ of DMSO solution, and was transferred into a cellophane membrane dialysis bag (Jeong et al., 2006a). Then, the amphiphilic molecules of AA-CSP graft copolymers were dialyzed with $1.0 \mathrm{~L}$ of water each time and were dialyzed for eight times. Deionized water $(1.0 \mathrm{~L})$ was exchanged every 2 $\mathrm{h}$ for four times and then exchanged every $8 \mathrm{~h}$ for four times with $100 \mathrm{rpm} / \mathrm{min}$ at $25{ }^{\circ} \mathrm{C}$. The nanoparticle solution was filtered through a $0.45 \mathrm{~mm}$ membrane filter and was adjusted to $25 \mathrm{~mL}$ by adding deionized water. Docetaxel $(10 \mathrm{mg})$ was completely dissolved in $10 \mathrm{~mL}$ of chloroform/absolute ethanol mixture solvent $(1: 1, \mathrm{v} / \mathrm{v})$ and then slowly added into a nanoparticle solution drop by drop under magnetic stirring (100 rpm/min) for $24 \mathrm{~h}$. The harvested nanoparticle solution was adjusted to 25 $\mathrm{mL}$ by re-adding deionized water.

\section{Transmission electron microscopy}

The surface morphologies of the DTX-AA-CSP nanoparticles were observed on a transmission electron microscope (JEM-2010, Japan; Jaimuang, 2015). A droplet from the nanoparticles was stained with $0.5 \%$ phosphotungstic acid solution for $10 \mathrm{~min}$ and placed on a copper grid. Subsequently, the excess solution was removed by a filter paper. The sample was dried at room temperature before it was characterized. All the experiments were performed in triplicate.

\section{Zeta potentials and particle sizes}

The particle sizes and zeta potentials of the DTXAA-CSP nanoparticles were determined by a dynamic light scattering analyzer with a scattering angle of $90^{\circ}$ (Zetasizer Nano ZS, Malvern Instruments, UK) at $25^{\circ} \mathrm{C}$ (Guan et al., 2016a). All experiments were performed in triplicate, and data were expressed as mean value \pm standard deviations.

\section{Encapsulation efficiency and loading capacity}

The percentage drug loading efficiency of DTX in AA-CSP nanoparticles was determined by separating the unentrapped drug from nanoparticles by centrifugation at $12,000 \mathrm{rpm}$ for $10 \mathrm{~min}$. The clear supernatant was determined for the contents of DTX by measuring the peak area with a high performance liquid chromatography (HPLC) (LC-20AT, Shimadzu) at a wavelength of $230 \mathrm{~nm}$. The mobile phase consisted of distilled water and acetonitrile at a 45:55 volume ratio. All experiments were performed in triplicate. The percentage loading capacity and encapsulation efficiency were calculated as follows:

$\mathrm{EE}(\%)=\left(\mathrm{DTX}_{t}-\mathrm{DTX}_{f}\right) / \mathrm{DTX}_{t} * 100 \%$,

LC $(\%)=\left(\right.$ DTX $\left.\left._{t}-\mathrm{DTX}_{f}\right)\right) /$ Weight of copolymer micelles*100\%,

where DTXt was the total weight of docetaxel; and DTXf was the unentrapped docetaxel present in the supernatant.

\section{In vitro drug release}

The in vitro release profiles of DTX from docetaxel injection and DTX-AA-CSP nanoparticles were investigated through dialysis. DTX-AA-CSP nanoparticles and docetaxel injection were respectively suspended in $4 \mathrm{~mL}$ of distilled water $(100 \mu \mathrm{g} / \mathrm{mL}$ of final DTX concentration) and then transferred into a cellophane membrane dialysis bag (8-12 kDa). The dialysis bag was suspended in $20 \mathrm{~mL}$ of $\mathrm{pH} 6.8$ phosphate buffer saline (PBS) with $0.5 \%$ of Tween 80 under horizontal stirring at $100 \mathrm{rpm} / \mathrm{min}$ and temperature maintained at $37 \pm 0.5^{\circ} \mathrm{C}$ (Yanasarn, Sloat, Cui, 2009). A sample medium (5 mL) was withdrawn at predetermined time points $(0,1,2,4,6,8,10,12$, 24 , and $48 \mathrm{~h}$ ) and was compensated an equal volume of fresh PBS medium under the same temperature. The content of DTX in the released medium was calculated by HPLC at $230 \mathrm{~nm}$. Sink condition was maintained during the whole release period. All samples were performed in triplicate.

\section{Biocompatibility study of AA-CSP nanoparticles}

The biocompatibility of delivery carriers (AA-CSP nanoparticles) was analyzed via MTT assay29 using HUVEC cells. Cells were grown in 24-well plates at an initial density of $2 \times 105$ cells/well with $100 \mu \mathrm{L}$ of F12K containing $10 \%$ FBS, $0.4 \%$ heparin sodium, and $1 \%$ endothelial cell growth supplement, which were 
incubated for $24 \mathrm{~h}$ at $37{ }^{\circ} \mathrm{C}$ in a $5 \% \mathrm{CO}_{2}$ condition. The medium was placed by samples of various concentrations of AA-CSP nanoparticles $(0.05,0.2$, and $1 \mathrm{mg} / \mathrm{mL}$ ) and in pace with five paralleled wells. After $72 \mathrm{~h}$ of incubation, $20 \mu \mathrm{L}$ of MTT solution $(5 \mathrm{mg} /$ $\mathrm{mL}$ ) was placed to each well of the plate. The medium was discarded after $4 \mathrm{~h}$ of incubation, $200 \mu \mathrm{L} /$ well of DMSO was added to dissolve the formazan crystals in the plate, and the absorbance was measured at $492 \mathrm{~nm}$ by a microplate reader (FL600, Bio-Tek Inc., Winooski, VT). The cell viability (\%) was calculated according to the following equation:

Cell viability $(\%)=\left(\mathrm{OD}_{492, \text { sample }}-\mathrm{OD}_{492, \text { blank }}\right) /\left(\mathrm{OD}_{492, \text { control }}\right.$ $\left.-\mathrm{OD}_{492, \text { blank }}\right) \times 100$,

where OD492, sample represents the measurement from the AA-CSP nanoparticles, docetaxel injection (Taxotere $\left.{ }^{\circledR}\right)$; and DTX-AA-CSP nanoparticles; OD492, control represents from the cells treated with incubated solution; and OD492, blank represents the incubated solution.

\section{In vitro anticancer activity}

The anticancer activities of AA-CSP nanoparticles, docetaxel injection (Taxotere ${ }^{\circledR}$ ), and DTX-AA-CSP nanoparticles were measured in HepG2 and SW480 cells using the MTT (3-[4,5-dimethylthiazol-2-yl]-2,5diphenyl tetrazoniumbromide) assay (Mosmann, 1983). Briefly, SW480 and HepG2 cells with $2 \times 105$ cells/well of initial density were placed in a 96-well plate with 100 $\mu \mathrm{L}$ of DMEM containing $10 \%$ FBS and were incubated for $24 \mathrm{~h}$ at $37{ }^{\circ} \mathrm{C}$ in a $5 \% \mathrm{CO}_{2}$ condition. Cells were exposed in a series of doses of AA-CSP nanoparticles, docetaxel injection (Taxotere ${ }^{\circledR}$ ), and DTX-AA-CSP nanoparticles at concentrations ranging from $0.0005 \mu \mathrm{g} /$ $\mathrm{mL}$ to $0.5 \mu \mathrm{g} / \mathrm{mL}$. After $72 \mathrm{~h}$ of incubation, $20 \mu \mathrm{L}$ of MTT solution $(5 \mathrm{mg} / \mathrm{mL})$ was placed on each well of the plate. The medium was discarded after incubating for $4 \mathrm{~h}, 200 \mu \mathrm{L} /$ well of DMSO was added to dissolve the formazan crystals in the plate, and the absorbance was measured at $492 \mathrm{~nm}$ by a microplate reader (FL600, Bio-Tek Inc., Winooski, VT). The anticancer activity (\%) was calculated according to the following equation:

Anticancer activity $(\%)=\left(\mathrm{OD}_{492, \text { control }}-\mathrm{OD}_{492, \text { sample }}\right) /$ $\left(\mathrm{OD}_{492, \text { control }}-\mathrm{OD}_{492, \text { blank }}\right) \times 100$, where the OD492, sample represents the measurement from the AA-CSP nanoparticles, docetaxel injection (Taxotere ${ }^{\circledR}$ ), and DTX-AA-CSP nanoparticles; OD492, control represents the cells treated with incubated solution; and OD492 blank represents the incubated solution.

\section{Data analysis}

Data were expressed as the mean \pm standard deviation, and statistical analysis was performed using an unpaired Student's t-test. Data were considered significantly different at $\mathrm{p}<0.05$.

\section{RESULTS AND DISCUSSION}

\section{FT-IR and ${ }^{1} \mathrm{H}-\mathrm{NMR}$ Analysis}

FT-IR spectra of CSP and AA-CSP were shown in Figure 3. Compared with the spectrum of CSP, an obvious change was observed at $1730 \mathrm{~cm}^{-1}$, which resembled the existence of the (-OCO-group), and the result demonstrated that AA was successfully conjugated with CSP during the reaction. The broad stretching peak at $3435 \mathrm{~cm}^{-1}$ was attributed to hydroxyl groups, and the sharp peak near $1413 \mathrm{~cm}^{-1}$, to $\mathrm{O}-\mathrm{H}$ bending vibration. The band at $1640 \mathrm{~cm}^{-1}$ could be attributed to the ring stretching of glucose (Wang, 2011). The characteristic peak at $1085 \mathrm{~cm}^{-1}$ suggested the existence of pyran-glycosylated, and the absorption peak at $825 \mathrm{~cm}^{-1}$ suggested the presence of the $\alpha$-glycosidic linkage. The FT-IR spectrum of CSP and the above composition analysis indicated that CSP was mainly composed of $\alpha$-glycosidic, bond-linked glucopyranose.

Figures 4 and 5 showed the $1 \mathrm{H}$ NMR spectrum of CSP and AA-CSP in DMSO-d6, respectively, where $\delta 1.24 \mathrm{ppm}$ was the peak of methylene protons, and $\delta 0.85 \mathrm{ppm}$ was the peak of methyl protons. Hydroxyl proton signals were observed at $\delta 3.89-5.15 \mathrm{ppm}$ in the 1H NMR spectrum of CSP. $\delta 5.15$ ppm was the peak area of $(1 \rightarrow 6)$-linked hydrogen protons, and $\delta 4.91 \mathrm{ppm}$ was the peak area of $(1 \rightarrow 4)$-linked hydrogen proton in CSP(Cheong, 2016). Furthermore, the DS of AA-CSP was $13.88 \%$, which could be calculated from the peak areas of their 1H NMR signals, respectively (Table I). In this study, CSP was substituted by 13.88 stearic acid groups per 100 glucoside units. 


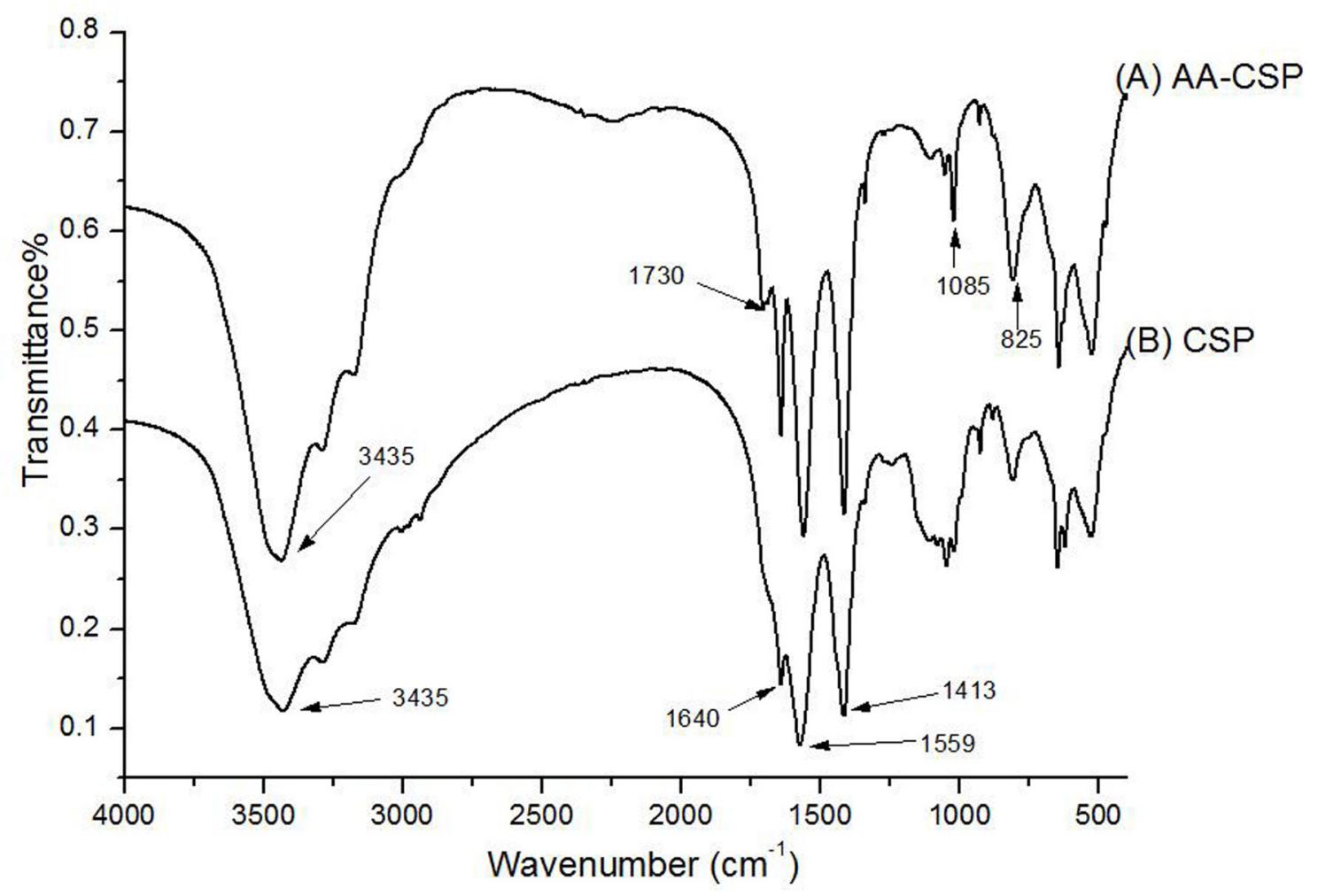

FIGURE 3 - Fourier transform infrared (FT-IR) spectra of AA-CSP (A) and CSP (B). FTIR experiments were recorded at $25^{\circ} \mathrm{C}$ on Shimadzu 8300 FTIR spectrometer with a KBr tablet with the range of $400-4000 \mathrm{~cm}^{-1}$.

\section{C. sinensis polysaccharides(CSP)}

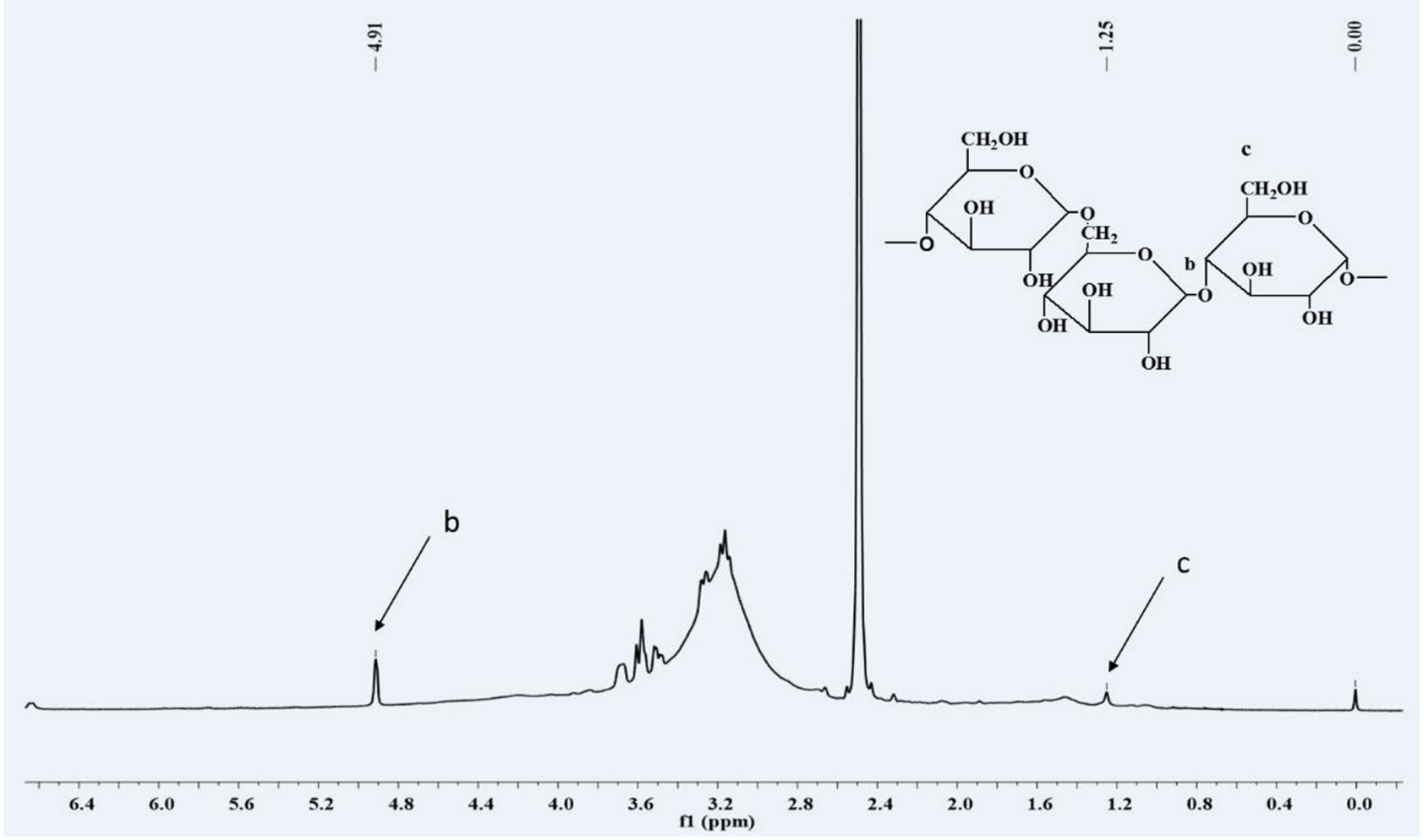

FIGURE 4 - ${ }^{1} \mathrm{H}$ nuclear magnetic resonance ( $\left.{ }^{1} \mathrm{H}-\mathrm{NMR}\right)$ spectra of CSP in $\mathrm{DMSO}_{d 6}$. 


\section{Acetic acid conjugated $C$. sinensis polysaccharides(AA-CSP)}

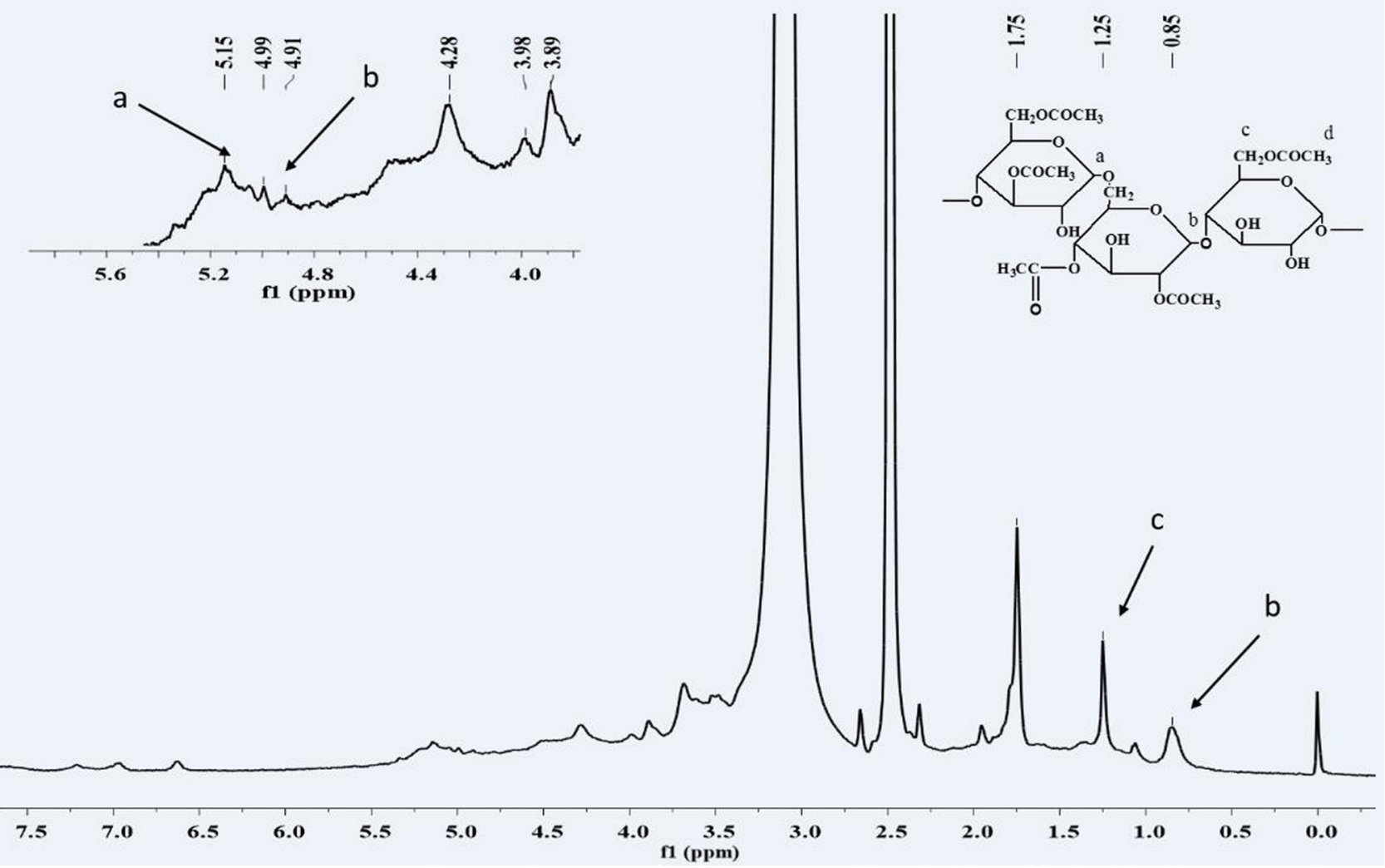

FIGURE $5-{ }^{1} \mathrm{H}$ nuclear magnetic resonance $\left({ }^{1} \mathrm{H}-\mathrm{NMR}\right)$ spectra of AA-CSP in $\mathrm{DMSO}_{d 6} \cdot{ }^{1} \mathrm{H}-\mathrm{NMR}$ spectra indicated the generation of methyl $\left(\delta_{0.85}\right)$ protons after the addition of AA to the reaction mixture containing CSP.

TABLE I - Peak area of $\delta 5.15, \delta 4.91, \delta 0.851 \mathrm{H}$ NMR signals

\begin{tabular}{lccc}
\hline Sample & $\mathbf{A}_{85.15}$ & $\mathbf{A}_{84.91}$ & $\mathbf{A}_{80.85}$ \\
\hline$A A-C S P$ & 1.01 & 1.40 & 1.00 \\
\hline
\end{tabular}

\section{Morphology analysis, zeta potentials, and particle sizes}

Amphiphilic copolymers, such as hydrophilic polysaccharides and hydrophobic groups, formed nanosized carriers with an outer hydrophilic shell and inner hydrophobic core structure in an aqueous medium (Jeong et al., 2006b). The special property of amphiphilic nanoparticles is suitable to incorporate and load poorly soluble anticancer drugs. The morphology of DTX-AA-CSP nanoparticles was characterized by a negative stain on the TEM. The nanoparticles had a spherical morphology (Figure 6).
The zeta potential of AA-CSP nanoparticles was $61.36 \pm 1.15 \mathrm{mV}$, whereas the average size was $-19.91 \pm 0.75$ $\mathrm{nm}$. The mean zeta potential and the particle size of DTX-AA-CSP nanoparticles were $98.91 \pm 0.29 \mathrm{~nm}$ and $-19.75 \pm 1.13 \mathrm{mV}$, respectively (Table II). The small particle size of nanoparticles $(<200 \mathrm{~nm})$ facilitated lymphatic transport, avoiding the mononuclear phagocyte system uptake, and passively delivering the anticancer drugs (Yokoyama et al., 1990). Zeta potential is also an important parameter, which reflects either the electrostatic repulsion or congregation of the nanoparticles. Increase in electrostatic repulsive force between nanoparticles can prevent coalescence of the nanoparticles from forming large precipitation, which is useful in maintaining the dispersion stability of these nanoparticles. Compared with that of blank AA-CSP nanoparticles, the mean particle size slightly increased, whereas zeta potential only changed slightly after DTX loading, when the DTX and AA-CSP nanoparticles mass ratio changed from 1:20 to 1:10 (w/w). 


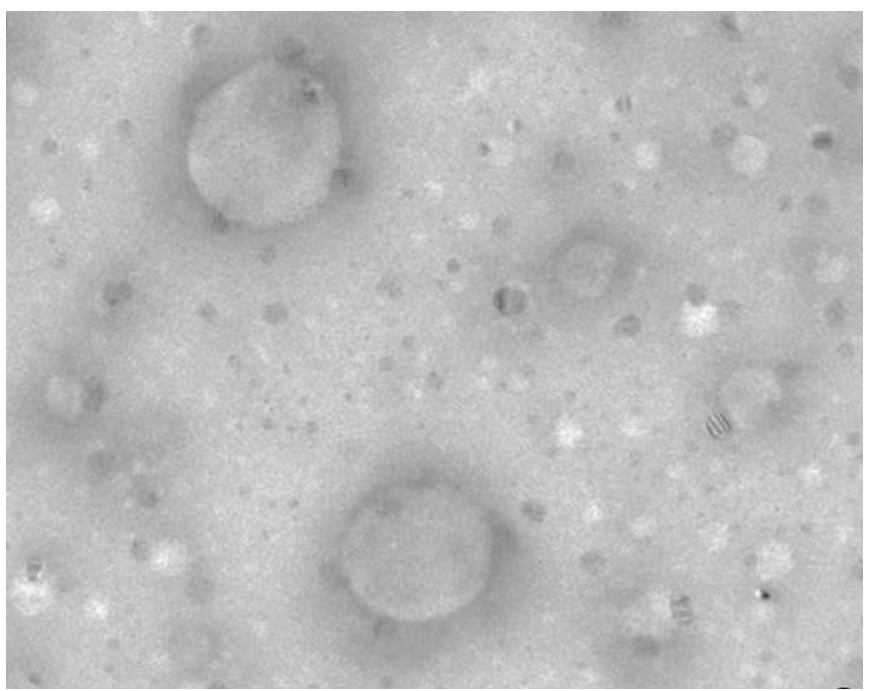

FIGURE 6 - Transmission electron microscopy image of DTX-AA-CSP nanoparticles (Magnitude 60000×,scale $200 \mathrm{~nm}$ ).

TABLE II - The characterization of DTX-AA-CSP nanoparticles

\begin{tabular}{cccccc}
\hline Sample & $\mathbf{d} / \mathbf{c}(\mathbf{w} / \mathbf{w})^{\mathbf{a}}$ & $\mathbf{E E}(\%)$ & $\mathbf{L C}(\mathbf{\%})$ & Average diameter $(\mathbf{n m})$ & Zeta potential $(\mathbf{m V})$ \\
\hline & -- & -- & -- & $61.36 \pm 1.15$ & $-19.91 \pm 0.75$ \\
& $1: 20$ & $85.87 \pm 0.57$ & $4.29 \pm 0.03$ & $86.40 \pm 1.20$ & $-21.05 \pm 0.82$ \\
AA-CSP & $1: 15$ & $83.55 \pm 0.50$ & $5.57 \pm 0.03$ & $97.70 \pm 1.02$ & $-21.44 \pm 0.52$ \\
& $1: 10^{\mathbf{b}}$ & $80.95 \pm 0.43$ & $8.09 \pm 0.04$ & $98.91 \pm 0.29$ & $-19.75 \pm 1.13$ \\
& $1: 9$ & $68.97 \pm 0.24$ & $7.66 \pm 0.03$ & $91.11 \pm 0.80$ & $-20.06 \pm 0.16$ \\
\hline
\end{tabular}

a The weight of drug and carrier(DTX/AA-CSP) self-aggregated nanoparticles (mg/mg)

${ }^{b}$ The optimized formulation.

\section{Encapsulation efficiency and loading capacity}

Table II also showed the effect of different counts of DTX on the LC and EE. Results presented that EE decreased from $85.87 \% \pm 0.57 \%$ to $80.95 \% \pm 0.50 \%$ with the increase of drug verse carrier mass ratio from $1: 20$ to $1: 10(\mathrm{w} / \mathrm{w})$ in this investigation. However, the LC (from $4.29 \% \pm 0.03 \%$ to $8.09 \% \pm 0.04 \%$ ) increased with the mass ratio ranging from 1:20 to $1: 10$, which indicated that the DTX-loading ability of AA-CSP nanoparticles was enhanced. The drug LC and EE of
DTX-AA-CSP nanoparticles were $8.09 \% \pm 0.04 \%$ and $80.95 \% \pm 0.43 \%$, respectively, when the drug verse carrier mass ratio was 1:10.

When the mass ratio of the drug to carrier was $1: 9$, the values of the LC and EE of DTX-AA-CSP nanoparticles decreased compared with that when the drug to carrier ratio was $1: 10$. The decreased value of LC and EE of the nanoparticles may have resulted from the capacity of AA-CSP themselves. The solvent polarity of chloroform/ethanol was used to dissolve DTX in the emulsion method. Chloroform/ethanol was 
liable in carrying DTX to enter the hydrophobic cores of AA-CSP nanoparticles. Therefore, considering the LC and $\mathrm{EE}$, we selected the mass ratio of drug verse carrier at 1:10 to prepare the DTX-AA-CSP nanoparticles, with the chloroform/ethanol as solvent to dissolve DTX. The optimized formulation was repeated in triplicate.

\section{In vitro drug release}

The release behavior of docetaxel injection and DTX-AA-CSP nanoparticles in vitro was performed in the phosphate buffer saline ( $\mathrm{pH}$ 6.8) solution containing $0.8 \%$ of Tween 80 at $37 \pm 0.5^{\circ} \mathrm{C}$. As shown in Figure 7, the release percentage of DTX from docetaxel injection Taxotere ${ }^{\circledR}$ was faster and higher $(77.55 \% \pm 0.89 \%)$ than that from DTX-AA-CSP nanoparticles $(54.17 \% \pm 1.08 \%)$ at $12 \mathrm{~h}$. The DTX-AA-CSP nanoparticles tended to be stable even after $12 \mathrm{~h}$. The DTX release percent of DTXAA-CSP nanoparticles was $61.94 \% \pm 1.45 \%$ in the first $24 \mathrm{~h}$ and $68.10 \% \pm 0.71 \%$ in $48 \mathrm{~h}$. The release behavior of DTX-AA-CSP nanoparticles was lower and slower than that of docetaxel injection Taxotere ${ }^{\circledR}$ at the same dissolution medium. The difference in DTX release rate was mainly due to the core-shell structure of AA-CSP nanoparticles.
The drug release from nanoparticles is related to their chemical and physical properties, such as partition coefficient between the drug and hydrophobic group, drug diffusion rate, and copolymer degradation (Sutthasupa, Sanda, 2016). These results suggested that DTX was gradually released from the DTX-AA-CSP nanoparticles, and a slower release rate was maintained for a relatively longer time.

\section{Biocompatibility study of the delivery carrier (AA-CSP nanoparticles)}

MTT assay was also analyzed on HUVEC to investigate the biocompatibility of AA-CSP nanoparticles toward normal cells. As shown in Figure 8 , with concentrations of AA-CSP nanoparticles ranging from $0.05 \mathrm{mg} / \mathrm{mL}$ to $1 \mathrm{mg} / \mathrm{mL}$, the cell viability of HUVEC cells were all above $90 \%$, which revealed that the nanoparticles were safe to be used as drug carriers below $1 \mathrm{mg} / \mathrm{mL}$. According to previous literature, the DTX therapeutic concentration was $6.45 \pm 1.18 \mu \mathrm{g} / \mathrm{mL}$ after intravenous infusion at $1 \mathrm{~h}$ at a $75 \mathrm{mg} / \mathrm{m}^{2}$ dose. The corresponding carrier material (AA-CSP nanoparticles) concentration was $43.58 \pm 7.97 \mu \mathrm{g} / \mathrm{mL}$ (Guan et al., 2016 $\mathrm{a}$ or b???). Toxicity results indicated that AA-CSP

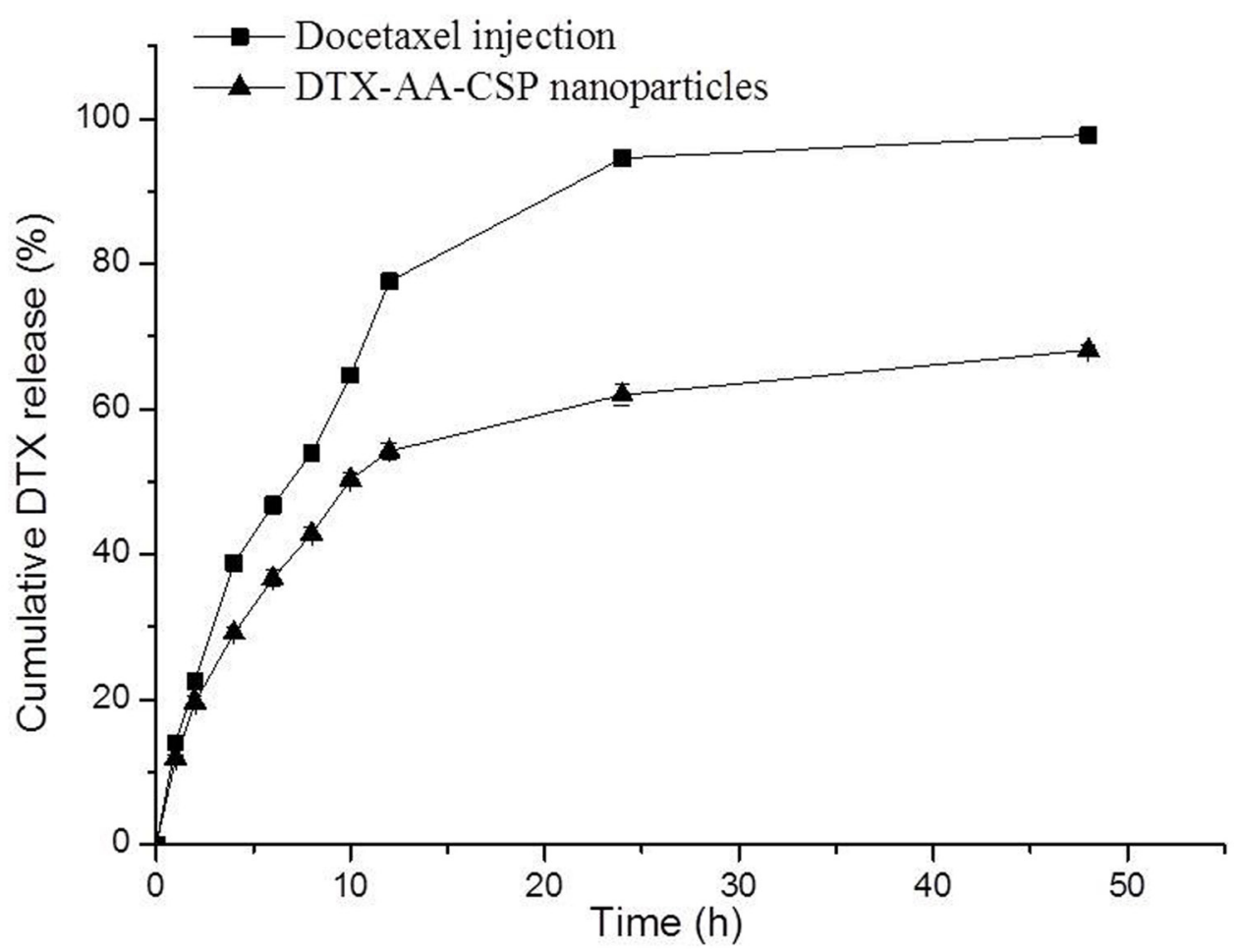

FIGURE 7 - In vitro release profiles of DTX from docetaxel injection (-口-) and DTX-AA-CSP nanoparticles (- $\mathbf{\Delta}$-) in pH 6.8 phosphate-buffered saline containing $0.5 \%$ of Tween 80 at $37 \pm 0.5^{\circ} \mathrm{C}$. 
nanoparticles caused no toxicity below $1 \mathrm{mg} / \mathrm{mL}$. Thus, delivery carriers (AA-CSP nanoparticles) were safe and biocompatible, which can be used as a promising drug delivery system for antitumor drugs.

\section{In vitro anticancer activity}

The in vitro anticancer activity of the AA-CSP nanoparticles, docetaxel injection and DTX-AA-CSP nanoparticles was evaluated via the MTT assay using SW480 and HepG2 as a model liver cancer cell line. As shown in Figures 9 and 10, docetaxel injection and DTX-AA-CSP nanoparticles apparently had dosedependent inhibition against HepG2 and SW480 cells at an equivalent DTX dose from $0.0005 \mu \mathrm{g} / \mathrm{mL}$ to $0.5 \mu \mathrm{g} /$ mL. AA-CSP nanoparticles, Taxotere ${ }^{\circledR}$, and DTX-AACSP nanoparticles presented similar antitumor activity against HepG2 and SW480 cancer cells.

When AA-CSP nanoparticles concentration was $0.5 \mu \mathrm{g} / \mathrm{mL}$, the anticancer activity of blank AACSP nanoparticles on SW480 and HepG2 cells was $22.52 \% \pm 2.03 \%$ and $22.56 \% \pm 1.19 \%$, respectively. The blank AA-CSP copolymer micelles were still biocompatible, and AA-CSP showed less inhibition on cancer cell growth. When DTX concentration was $0.5 \mu \mathrm{g} / \mathrm{mL}$, the antitumor activities of DTX-AA-CSP nanoparticles and docetaxel injection on SW480 and
HepG2 cells were $52.96 \% \pm 1.43 \%$ and $43.62 \% \pm 0.95 \%$, respectively, whereas the anticancer activity was $48.48 \% \pm 0.57 \%$ and $40.92 \% \pm 2.86 \%$ at $0.5 \mu \mathrm{g} / \mathrm{mL}$ drug concentration, respectively. The anticancer activities of DTX-AA-CSP nanoparticles against HepG2 and SW480 cancer cells were stronger than those of Taxotere ${ }^{\circledR}$. Results also revealed that DTX-AA-CSP nanoparticles were highly effective against tumor cells and improved the tumor therapy of free DTX.

One possible reason for enhanced tumor therapy was the property of nanoparticles. Drug-loaded nanoparticles have excellent dispersion, large specific surface area, and small particle diameter. It is beneficial for the improvement of the drug-loaded effect due to its large surface energy and chemical activity. At the same time, the good compatibility of this nanoparticle is favorable to enter cancer cells through endocytosis. Nanoparticles with an approximately $100 \mathrm{~nm}$ diameter improved tumoral uptake by 10-20 folds compared with that of other diameters due to its good permeability and retention (Moreira, Gaspar, Allen, 2001). DTX-AACSP nanoparticles can take DTX into the cancer cells through endocytosis and increase intracellular DTX accumulation (Wong et al., 2006). Cancer cells have a particular endocytic route and internalize DTX-AA-CSP nanoparticles into the interior of the cell, which increase drug concentration near the action site (Morrisa et al.,

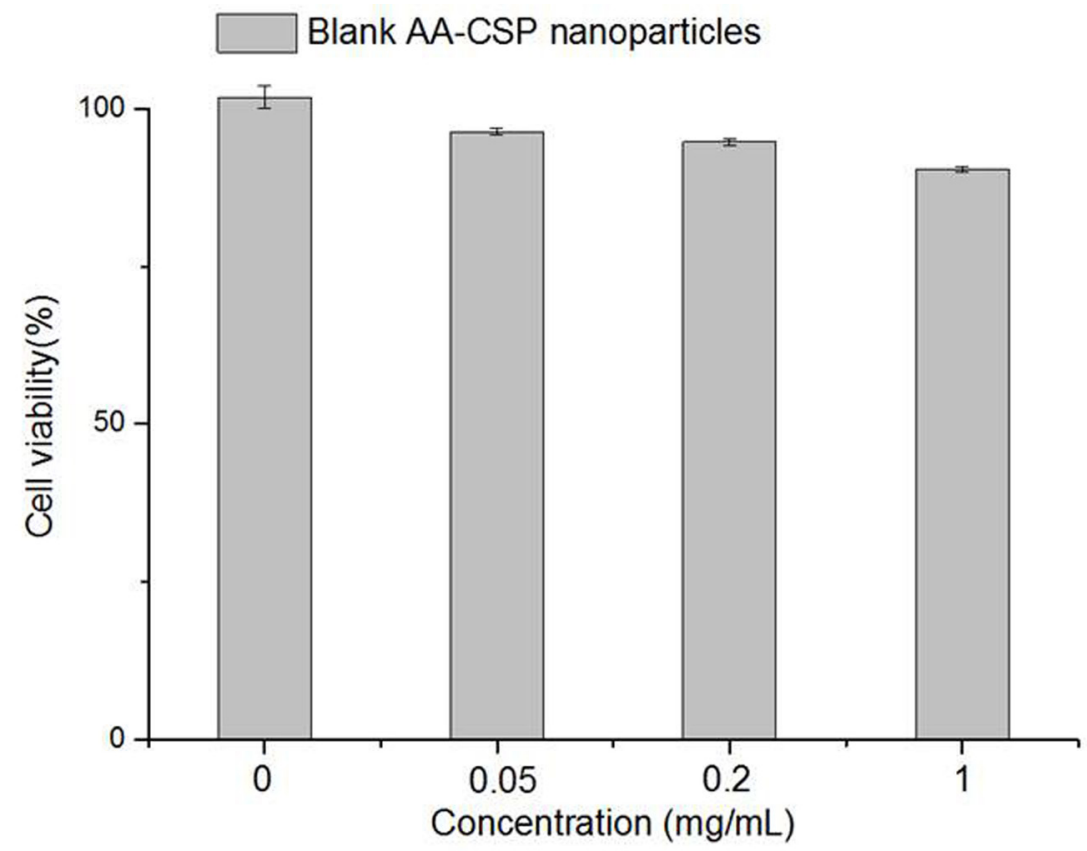

FIGURE 8 - Biocompatibility measurement of AA-CSP nanoparticles on HUVEC cells. 


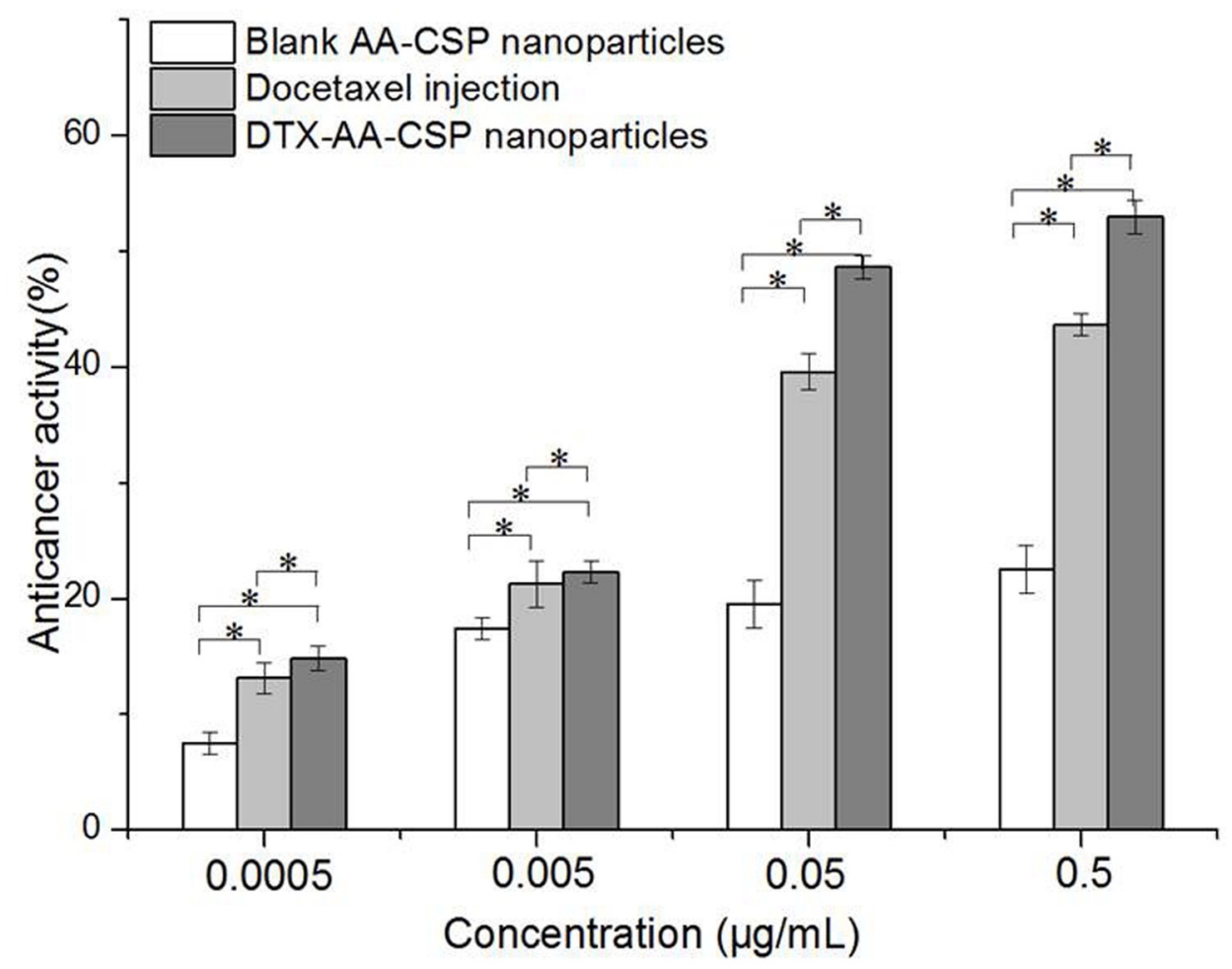

FIGURE 9 - Anticancer activity of AA-CSP nanoparticles, docetaxel injection (Taxotere ${ }^{\circledR}$ ) and DTX- AA-CSP nanoparticles on SW 480 cells after $48 \mathrm{~h}$ incubation $(* p<0.05)$.

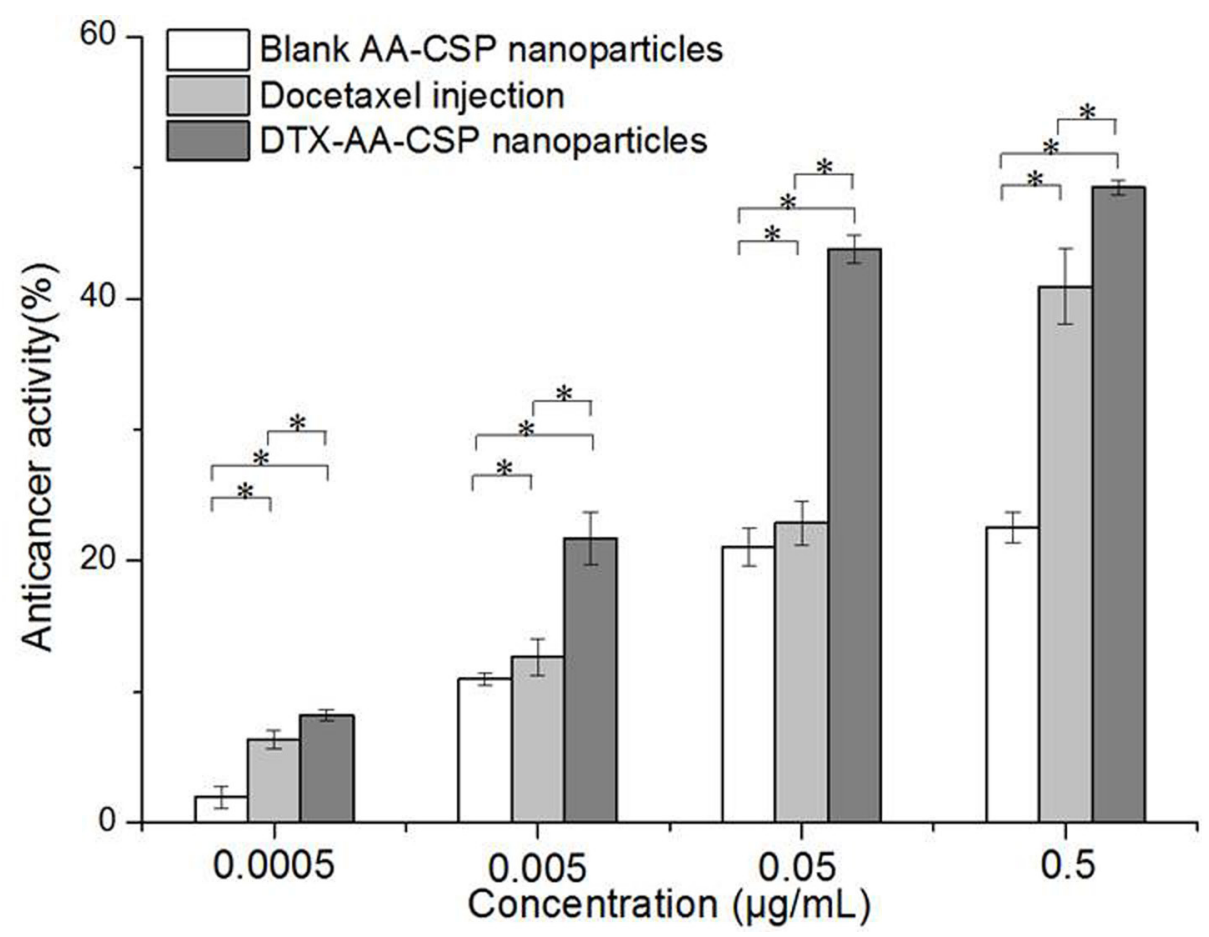

FIGURE 10 - Anticancer activity of AA-CSP nanoparticles, docetaxel injection (Taxotere ${ }^{\circledR}$ ) and DTX-AA-CSP nanoparticles on HepG2 cells after $48 \mathrm{~h}$ incubation $(* p<0.05)$. 
2011). In addition, DTX-AA-CSP nanoparticles probably avoid the DTX efflux effect by inhibiting drug efflux pumps (e.g. P-glycoprotein [P-gp]), which commonly exist in many tumor cells with multidrug resistance. However, the intrinsic mechanism of improving tumor therapy for DTX-AA-CSP nanoparticles will need further investigation in our next work.

\section{CONCLUSION}

In this study, a novel amphiphilic copolymer AACSP was synthesized through the covalent attachment of AA to CSP derived from TCM and was characterized by $1 \mathrm{H}-\mathrm{NMR}$ and FT-IR. The copolymer can easily self-assemble into micelle nanoparticles in an aqueous medium. DTX-AA-CSP nanoparticles had a particle diameter of $98.91 \pm 0.29 \mathrm{~nm}$, LC of $8.09 \% \pm 0.04 \%$, and EE of $80.95 \% \pm 0.43 \%$. The DTX-AA-CSP nanoparticles maintained a relatively longer release time than the marketed DTX injection, Taxotere ${ }^{\circledR}$. AA-CSP nanoparticles had good biocompatibility and no toxicity at therapeutic concentration toward HUVEC cells. The anticancer activity of DTX-AA-CSP nanoparticles against HepG2 and SW480 cells was superior to that of Taxotere ${ }^{\circledR}$. Docetaxel injection and DTX-AA-CSP nanoparticles apparently inhibit HepG2 and SW480 cells in a dose-dependent manner. This work is beneficial to the development of polymer micelle nanoparticles based on TCM and provides a promising biocompatible delivery system (AA-CSP nanoparticle) to carry hydrophobic antitumor drugs for cancer therapy.

\section{REFERENCES}

Agnihotri SA, Mallikarjuna NN, Aminabhavi TM. Recent advances on chitosan-based micro- and nanoparticles in drug delivery. J Control Release. 2004;100(1):5-28.

Cheong KL, Chen XQ, Meng LZ, Wang LY, Wu DT, Zhao J, Li SP. Structural elucidation, chain conformation and immuno-modulatory activity of glucogalactomannan from cultured Cordyceps sinensis fungus UM01. J Funct Foods. 2016;25:174-185.

Cheung JK, Li J, Cheung AW, Zhu Y, Zheng KY, Bi CW, Duan R, Choi RC, Lau DT, Dong TT, Lau BW, Tsim KW. Cordysinocan, a polysaccharide isolated from cultured Cordyceps, activates immune responses in cultured T-lymphocytes and macrophages: signaling cascade and induction of cytokines. J Ethnopharmacol. 2009;124(1):61-68.
Feeney OM, Crum MF, McEvoy CL, Trevaskis NL, Williams HD, Pouton CW, Charman WN, Bergström CAS, Porter CJH. 50 years of oral lipid-based formulations: Provenance, progress and future perspectives. Adv Drug Deliv Rev. 2016;101:167-194.

Guan Q, Sun D, Zhang G, Sun C, Wang M, Ji DY, Yang W. Docetaxel-loaded self-assembly stearic acid-modified bletilla striata polysaccharide micelles and their anticancer effect: Preparation, characterization, cellular uptake and in vitro evaluation. Molecules. 2016a;21(12):E1641.

Guan Q, Zhang G, Sun S, Fan H, Sun C, Zhang S. Enhanced oral bioavailability of Pueraria Flavones by a novel solid self-microemulsifying drug delivery system (SMEDDS) Dropping Pills. Bio Pharm Bull. 2016b;39(5):762-769.

Jaimuang S, Vatanatham T, Limtrakul S, Prapainainar P. Kinetic studies of styrene-grafted natural rubber emulsion copolymerization using transmission electron microscope and thermal gravimetric analysis. Polymer. 2015;67:249-257.

Jeong YI, Kim SH, Jung TY, Kim IY, Kang SS, Jin YH, Ryu HH, Sun HS, Jin S, Kim KK, Ahn KY, Jung S. Polyion complex micelles composed of all-trans retinoic acid and poly (ethylene glycol)-grafted-chitosan. J Pharm Sci. 2006a;95(11):2348-2360.

Jeong YI, Na HS, Oh JS, Choi KC, Song CE, Lee HC. Adriamycin release from self-assembling nanospheres of poly(DL-lactide-co-glycolide)-grafted pullulan. Int J Pharm. 2006b;322(1-2):154-160.

Kamel S, Ali N, Jahangir. K, Shah SM, El-gendy AA. Pharmaceutical significance of cellulose: A review. Express Poly Lett. 2008;2(11):758-778.

Lee KY, Jo WH, Kwon IC, Kim AY, Jeong SY. Structural determination and interior polarity of self-aggregates prepared from deoxycholic acid-modified chitosan in water. Macromolecules. 1998;31(2):378-383.

Li SP, Zhang GH, Zeng Q, Huang ZG, Wang YT, Dong TT, Tsim KW. Hypoglycemic activity of polysaccharide, with antioxidation, isolated from cultured Cordyceps mycelia. Phytomedicine. 2006;13(6):428-433.

Li SP, Zhao KJ, Ji ZN, Song ZH, Dong TT, Lo CK, Cheung JK, Zhu SQ, Tsim KW. A polysaccharide isolated from Cordyceps sinensis, a traditional Chinese medicine, protects PC12 cells against hydrogen peroxide-induced injury. Life Sci. 2003;73(19):2503-2513.

Liao YH, Jones SA, Forbes B, Martin GP, Brown MB. Hyaluronan: pharmaceutical characterization and drug delivery. Drug delivery. 2005;12(6):327-342 . 
McHale R, Patterson JP, Zetterlund PB, O’Reilly RK. Biomimetic radical polymerization via cooperative assembly of segregating templates. Nat Chem. 2012;4(6):491-497. Nat Chem. 2012;4(6):491-497.

Mosmann T. Rapid colorimetric assay for cellular growth and survival_ Application to proliferation and cytotoxicity assays. J Immunol Methods. 1983;65(1-2):55-63.

Moreira JN, Gaspar R, Allen TM. Targeting Stealth liposomes in a murine model of human small cell lung cancer. Biochim Biophys Acta. 2001;1515(2):167-176.

Morrisa GA, Castile J, Smith A, Adams GG, Harding SE. The effect of prolonged storage at different temperatures on the particle size distribution of tripolyphosphate (TPP)-chitosan nanoparticles. Carbohydr Polym. 2011;84(4):1430-1434.

Motozato Y, Ihara H, Tomoda T, Hirayama C. Preparation and gel permeation chromatographic properties of pullulan spheres. J Chromatogr A. 1986;355:434-437.

Nishikawa T, Akiyoshi K, Sunamoto J. Macromolecular complexation between bovine serum albumin and the self-assembled hydrogel nanoparticle of hydrophobized polysaccharides. J Am Chem Soc. 1996;118(26):6110-6115.

Pakulska MM, Miersch S, Shoichet MS. Designer protein delivery: From natural to engineered affinity-controlled release systems. Science 2016;351(6279):aac4750.

Pattni BS, Chupin VV, Torchilin VP. New Developments in liposomal drug delivery. Chem Rev. 2015;115(19):1093810966.

Powers AS, Liao HG, Raja SN, Bronstein ND, Alivisatos AP, Zheng $H$. Tracking nanoparticle diffusion and interaction during self-assembly in a liquid cell. Nano Lett. 2017;17(1):15-20.

Segal M, Avinery R, Buzhor M, Buzhor M, Shaharabani R, Harnoy AJ, Tirosh E, Beck R, Amir RJ. Molecular precision and enzymatic degradation: from readily to undegradable polymeric micelles by minor structural changes. J Am Chem Soc. 2017;139(2):803-810.

Shi J, Kantoff PW, Wooster R, Farokhzad OC. Cancer nanomedicine: progress, challenges and opportunities. Nat Rev Cancer. 2017;17(1):20-37.

Shi Y, Kim S, Huff TB, Borgens RB, Park K, Shi R, Cheng JX. Effective repair of traumatically injured spinal cord by nanoscale block copolymer micelles. Nat Nanotechnol. 2010;5(1):80-87.
Singh RS, Kaur N, Kennedy JF. Pullulan and pullulan derivatives as promising biomolecules for drug and gene targeting. Carbohydr Polym. 2015;123:190-207.

Sutthasupa S, Sanda F. Synthesis of diblock copolymers of indomethacin/aspartic acid conjugated norbornenes and characterization of their self-assembled nanostructures as drug carriers. Euro Polymer J. 2016:85:211-224.

Torchilin VP. Multifunctional, stimuli-sensitive nanoparticulate systems for drug delivery. Nat Rev Drug Discov. 2014;13(11):813-827.

Wang ZM, Peng X, Lee KLD, Tang Jco, Cheung PCK, Wu JY. Structural characterisation and immunomodulatory property of an acidic polysaccharide from mycelial culture of Cordyceps sinensis fungus Cs-HK1. Food Chem. 2011;125(2):637-643.

Wong HL, Bendayan R, Rauth AM, Xue HY, Babakhanian K, Wu XY. A mechanistic study of enhanced doxorubicin uptake and retention in multidrug resistant breast cancer cells using a polymer-lipid hybrid nanoparticle system. J Pharmacol Exp Ther. 2006;317(3):1372-1381.

Wu Y, Hu N, Pan Y, Zhou L, Zhou X. Isolation and characterization of a mannoglucan from edible Cordyceps sinensis mycelium. Carbohydrate Res. 2007;342(6):870-875.

Wu Y, Omar I, Sun C, Pa Y. Structure analysis and antitumor activity of (1-3)-beta-d-glucans (cordyglucans) from the mycelia of Cordyceps sinensis. Planta medica. 2005;71(4):381-384.

Xu H, Yao Q, Cai C, Gou J, Zhang Y, Zhong H, Tang X. Amphiphilic poly(amino acid) based micelles applied to drug delivery: the in vitro and in vivo challenges and the corresponding potential strategies. J Control Release. 2015;199:84-97.

Xie C, Xu N, Shao Y, He Y. Using FT-NIR spectroscopy technique to determine arginine content in fermented Cordyceps sinensis mycelium. Spectrochim Acta A Mol Biomol Spectrosc. 2015;149:971-977.

Yanasarn N, Sloat BR, Cui Z. Nanoparticles engineered from lecithin-in-water emulsions as a potential delivery system for docetaxel. Int J Pharm. 2009;379(1):174-180.

Yokoyama M, Miyauchi M, Yamada N, Okano T, Sakurai Y, Kataoka K, Inoue S. Characterization and anticancer activity of the micelle-forming polymeric anticancer drug adriamycin-conjugated poly (ethylene glycol)-poly (aspartic acid) block copolymer. Cancer Res. 1990;50(6):1693-1700.

Received for publication on $19^{\text {th }}$ June 2018 Accepted for publication on $23^{\text {rd }}$ September 2018 